\title{
Double imprinting in a single molecularly imprinted polymer format for the determination of ascorbic acid and dopamine
}

\section{Mahavir Prasad Tiwari, Rashmi Madhuri, Deepak Kumar, Darshika Jauhari, Bhim Bali Prasad}

\author{
Department of Chemistry, Faculty of Science, Banaras Hindu University, \\ Varanasi 221 005, India
}

*Corresponding author. Tel: (+91) 945-1954449; Fax: (+91) 542-22368127, E-mail: prof.bbpd@yahoo.com

Received: 25 Dec 2010, Revised: 04 March 2011 and Accepted: 08 March 2011

\section{ABSTRACT}

A new molecularly imprinted polymer - carbon composite fiber is constructed using reversible addition-fragmentation chain transfer polymerization technique. The fiber was evaluated as a sensor for the simultaneous determination of ascorbic acid and dopamine at ultratrace level, in aqueous samples, without any cross-reactivity. The binding characteristics of ascorbic acid and dopamine were also evaluated by differential pulse cathodic stripping voltammetry. Copyright (C) 2011 VBRI press.

Keywords: Molecularly imprinted polymer-carbon composite fiber; double imprinting, differential pulse cathodic stripping voltammetry; ascorbic acid and dopamine.
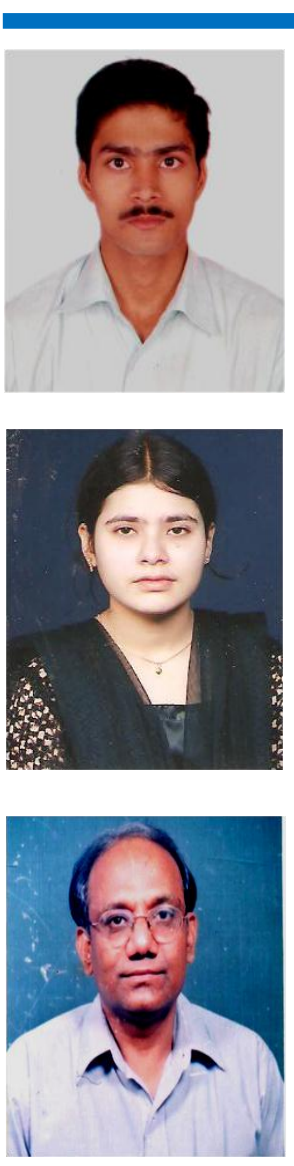

Mahavir Prasad Tiwari is a research scholar in Department of Chemistry, faculty of Science, BHU, India under the supervision of Dr. Bhim Bali Prasad. He received his B.Sc. in 2005 and M.Sc. in 2007 from Purvanchal University. His research interests lie in the field of solid phase extraction/microextraction, molecularly imprinted polymers, and electroanalytical chemistry.

Rashmi Madhuri is currently pursuing a Ph.D. at Banaras Hindu University under the supervision of Dr. Bhim Bali Prasad. She received her B.Sc. in 2005 and M.Sc. in 2007 from Banaras Hindu University, India. She is recipient of CSIR NET Junior Research Fellowship and BHU research fellowship. Her research interests lie in the field of chemical sensor development, molecularly imprinted biomimetic polymers, and electroanalytical chemistry.

Bhim Bali Prasad is currently a professor at the Banaras Hindu University, India where he has mentored 20 Ph.D. students and published 90 research papers in several reputed international and national journals. He received his B.Sc. degree in chemistry in 1972 and M.Sc. degree in 1974 form Banaras Hindu University, India. He obtained his Ph.D. from the Department of Chemistry, faculty of Science, BHU, India with Dr. Lal Mohan Mukherjee. $\mathrm{He}$ is a recipient of several national awards for his research contribution in analytical chemistry. He also went to Ranbaxy Ltd., India for about one-and-half year and elaborated a protocol for pharmaceutical analysis, interfaced with several sophisticated instruments. His research interests include environmental chemistry, chromatography, electroanalysis, and detection principle for chemical analysis and development of biomimetic chemical sensor using molecularly imprinted polymers for clinical, pharmaceutical and biological analyses.

\section{Introduction}

Artificial molecular recognition elements prepared by imprinting techniques attract increasing attention due to their potentials to act as selective sorbents in analytical sample pretreatment, chemical sensing, drug delivery, catalysis, dedicated separations, and finally screening elements in drug discovery. In most of the accounts of molecular imprinting, a single template molecule is used to create specific binding sites in the molecularly imprinted polymer (MIP) motif. However, the imprinting process should not be limited to a single template, and several compounds can also be imprinted simultaneously in a single polymer format. The concept of double imprinting introduces an additional degree of freedom in the material design, because the combination of two different print molecules enables the creation of more suitable hollows for analyte re-inclusion, improved diffusion pathways, and different classes of compounds can be extracted, separated, assayed, detected, or otherwise analyzed at one time $[\mathbf{1 , 2}]$. In this work, we have fabricated a monolithic fiber of MIP- 
carbon composite, where the non-conducting MIP layer was changed to be conducting, in the presence of carbon particles (dispersed as organized strip). Unlike the general MIP-composite electrode preparation protocol [3], we have resorted to a fresh approach for an in-situ synthesis of MIPcarbon composite fiber via reversible additionfragmentation chain transfer (RAFT) polymerization technique. RAFT has recently been emerged as a promising technique due to its versatility and simplicity, and the product (polymer) is free from the contamination of metal catalyst [4]. RAFT is a degenerative chain transfer free radical polymerization process. Such living radical polymerization technique is reportedly known to be creating homogeneous binding sites (cavities) within the MIP network.

The proposed MIP-carbon composite electrode was examined for the simultaneous determination of ascorbic acid (AA) and dopamine (DA), in aqueous samples using differential pulse cathodic stripping voltammetry (DPCSV). AA is a soluble vitamin present in many biological systems and in multivitamin preparations, which are commonly being used as supplement to inadequate dietary intake and as anti-oxidants [5]. The low concentration of AA in human blood serum results in scurvy and hypovitaminosis. The prevalence and risk factors owing to hypovitaminosis $\mathrm{C}$, consequent upon low serum ascorbic acid level, cause clinical manifestations primarily of hemorrhagic syndrome, edema, stomatologic signs, arthritis, myalgia, anorexia, and depression. Dopamine (DA), a biogenic catecholamine, is among one of the neurotransmitters of central and peripheral nervous systems. Measurement of DA in biological samples is useful for the clinical diagnosis of chronically progressive neurodegenerative diseases, e.g. Parkinson's disease (PD), schizophrenia and Alzheimer's disorder, with low DA levels in serum. Therefore, it is essential to develop a simple and rapid method for the determination of these biological molecules in routine analysis. Although numerous methods have been used for the selective determination of AA [6-10] and DA [11-15] including high performance liquid chromatography $[6,11]$, capillary electrophoresis [7, 12], soli-phase extraction [8, 13], and electrochemical methods $[9,10,14,15]$, only a few electrochemical attempts have been made for the simultaneous determination of AA and DA [16-18]. However, consequent upon the overlap of their voltammetric responses, the severe electrode fouling, poor selectivity, and reproducibility associated with these methods [16-18] make simultaneous determination of AA and DA highly difficult. Furthermore, no MIP-based technique is, hitherto, reported for the simultaneous determination of AA and DA. The present work thus deals, for the first time, with the high selectivity and sensitive simultaneous determination of AA and DA.

\section{Experimental}

\section{Materials and reagents}

Ascorbic acid (AA), carbon powder $(1-2 \mu)$, and solvents dimethylsulphoxide (DMSO), methanol, and ethanol were purchased from Loba chemie pvt. Ltd. (Mumbai, India). Ethylene glycol dimethacrylate (EGDMA), dopamine (DA), and benzyl-N, N-diethyldithiocarbamate (BDC, iniferter) were supplied from Aldrich (Steinheim, Germany). All the reagents were of AR grade and used as received. Phosphate buffer solution (PBS) $(\mathrm{pH} \mathrm{7.8,} \mathrm{ionic}$ strength $0.1 \mathrm{M}$ ) was used as a supporting electrolyte. Standard stock solutions (500 $\mathrm{mg} \mathrm{L}^{-1}$ ) of DA and AA were prepared using deionized triple-distilled water.

\section{Apparatus}

Voltammetric measurements were carried out with a polarographic analyzer/stripping voltammeter [model 264 A, EG \& G Princeton Applied Research (PAR)] in conjunction with an electrode assembly (PAR model 303 A) and X-Y chart recorder (PAR model RE 0089) (Fig. 1 A). Herein a conventional three-electrode system was used where MIP-carbon composite fiber, platinum wire, and saturated $\mathrm{Ag} / \mathrm{AgCl}$ served as working, auxiliary, and reference electrodes, respectively. 1H NMR characterization was made with the help of JEOL AL 300 FT/NMR (Japan) spectrometer. Morphological images of MIP-carbon composite were recorded on a scanning electron microscope (SEM) (JEOL, JSM, model 840 A, Netherlands).

\section{Preparation of MIP-carbon composite}

The monomer, 2,4,6-trisacrylamido-1,3,5-triazine (TAT), was prepared and characterized following a known recipe [19]. Accordingly, monomer (TAT, $1.0 \mathrm{mmol}, 1.0 \mathrm{~mL}$ DMSO) and templates (AA and DA, $0.5 \mathrm{mmol}$ of each dissolved in 1.0 mL DMSO) solutions were mixed together, followed by the addition of EGDMA (5.0 mmol, 1.0 $\mathrm{mL})$.To this mixture iniferter (BDC, $0.5 \mathrm{~mL}$ ) and an optimized amount $(80 \mathrm{mg}$ ) of carbon powder were added and stirred for $10 \mathrm{~min}$ to obtain a homogeneous but slightly viscous solution. The whole content was degassed gently with $\mathrm{N}_{2}$ stream for $10 \mathrm{~min}$. Finally, this mixture was filled in glass capillaries (inner diameter $0.8 \mathrm{~mm}$, length $4.0 \mathrm{~cm}$ ) with the help of a syringe and tightly sealed their openings by teflon tape. The filled-in capillaries were then kept strictly in the horizontal position in an oven, where the polymerization mixture was cured in a UV chamber for 4-5 h. This helped shaping MIP-carbon composite (monolithic) fiber in which carbon particles were organized as strip, under the action of gravity. The fibers were gently pushed out of capillaries by an insertable wire of diameter just close to that of capillary. The templates were retrieved from these fibers by immersing into $\mathrm{H}_{2} \mathrm{O}$-methanol $(9: 1$, v/v) solution for $40 \mathrm{~min}$, with mechanical stirring of the eluent at $600 \mathrm{rpm}$, until no voltammetric response of the template was observed.

\section{Sensor fabrication and voltammetric procedure}

For the sensor fabrication, $5.0 \mathrm{~mm}$ MIP fiber (diameter 0.8 $\mathrm{mm}$ ) was inserted in another capillary (6 mm length), which was half-filled with an aqueous agarose solution $(0.2 \mathrm{~g}$, $10 \%, 60^{\circ} \mathrm{C}$ ). This way only $1.0 \mathrm{~mm}$ length of fiber was fixed inside the agarose (for an easy electrical contact) and remaining $4 \mathrm{~mm}$ fiber retained out of the capillary. The capillary was housed with one end in a micropipette tip for safe handling of the extruded fiber; while a copper wire was used at agarose end for the electrical connection (Fig. 1B). Electrochemical experiments were performed in a three- 
electrode cell containing $10 \mathrm{~mL}$ PBS (pH 7.8). All DPCSV runs were recorded after purging with $\mathrm{N}_{2}$ gas for $10 \mathrm{~min}$. After blank run, test solution was added into the cell for subsequent measurement. After analyte accumulation for 90 $\mathrm{s}$ at $+1.2 \mathrm{~V}$ vs. $\mathrm{Ag} / \mathrm{AgCl}$ and $15 \mathrm{~s}$ equilibration time, DPCSV runs were recorded in the potential range varying from +0.5 to $-1.2 \mathrm{~V}$ at a scan rate $10 \mathrm{mVs}^{-1}$, pulse amplitude $25 \mathrm{mV}$, and pulse width $50 \mathrm{~ms}$. All DPCSV runs for each concentration of test analyte were quantified using the method of standard addition [20]. The limit of detection $(L O D)$ was calculated by the standard procedure $[\mathbf{2 0}]$.

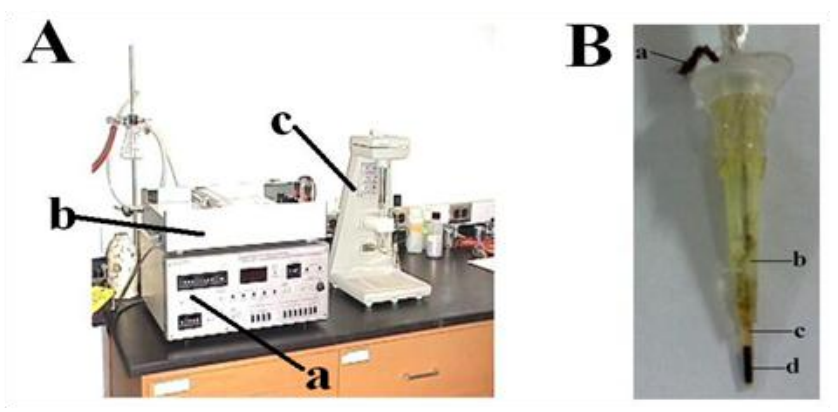

Fig. 1. (A) Apparatus for voltammetric measurement: (a) stripping voltammeter, (b) X-Y recorder, (c) three electrode assembly. (B) Picture of fabricated MIP-carbon composite fiber sensor: (a) copper wire, (b) micropipette tip, (c) agarose solution, and (d) MIP-carbon composite fiber.

\section{Results and discussion}

\section{Spectral characterization and recognition mechanism}

The rebinding of AA and DA in their respective molecular cavities, located at the surface or deeper inside the fiber textures, is represented in the structural diagram (Fig. 2). This revealed that the recognition occurred as a consequence of multiple hydrogen bonds. The proton NMR suggested binding interactions as follows: all AA peaks (meta, -OH), 6.2 (para, - $\mathrm{OH})$, and $\left.2.5\left(-\mathrm{NH}_{2}\right) \mathrm{ppm}\right]$ were shifted downfield to $11.4,3.1 \mathrm{ppm}$ (in case of AA) and 7.9, $6.25,3.3 \mathrm{ppm}$ (in case of DA), after binding with MIP. These peaks were simultaneously appeared and disappeared after rebinding and extraction, respectively. The porosity of the MIP fiber cross-section could easily be visualized from SEM (Fig. 3C). The black strip of ordered carbon particles can be seen in SEM (Fig. 3B) and even by the naked eye (Fig. 3A). MIP-carbon composite fiber sensors were obtained reproducibly with consistent thickness of $0.8 \mathrm{~mm}$, and with high endurance; these fibers were further regenerated by the method of template extraction for multiple measurements (more than 50 consecutive experiments).

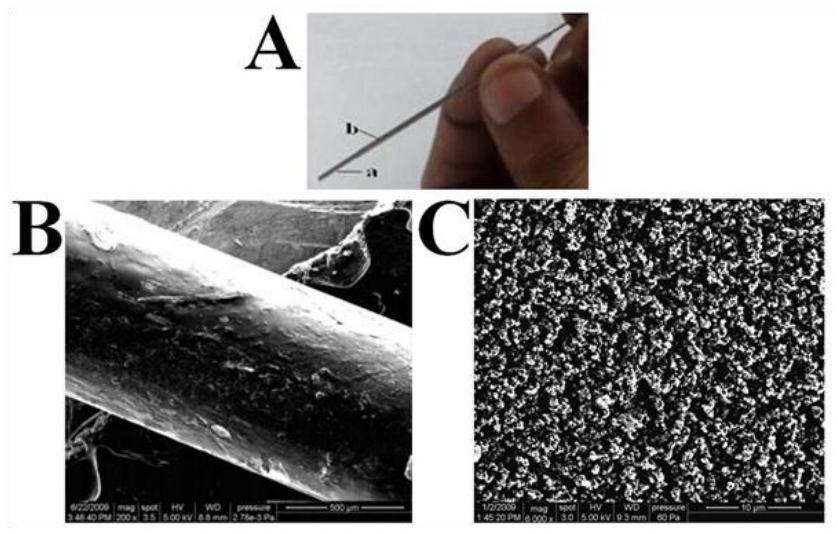

Fig. 3. (A) Picture of MIP-carbon composite fiber: (a) pushed out fiber with a visible black carbon strip, (b) glass capillary. (B) and (C) represent SEM images of MIP-carbon composite fiber at magnifications 200X and $6000 X$, respectively.

Simultaneous determination of $A A$ and $D A$ in aqueous medium

Typical DPCSV curves for the different concentrations of AA and DA demonstrated very smooth peaks (Fig. 4) both

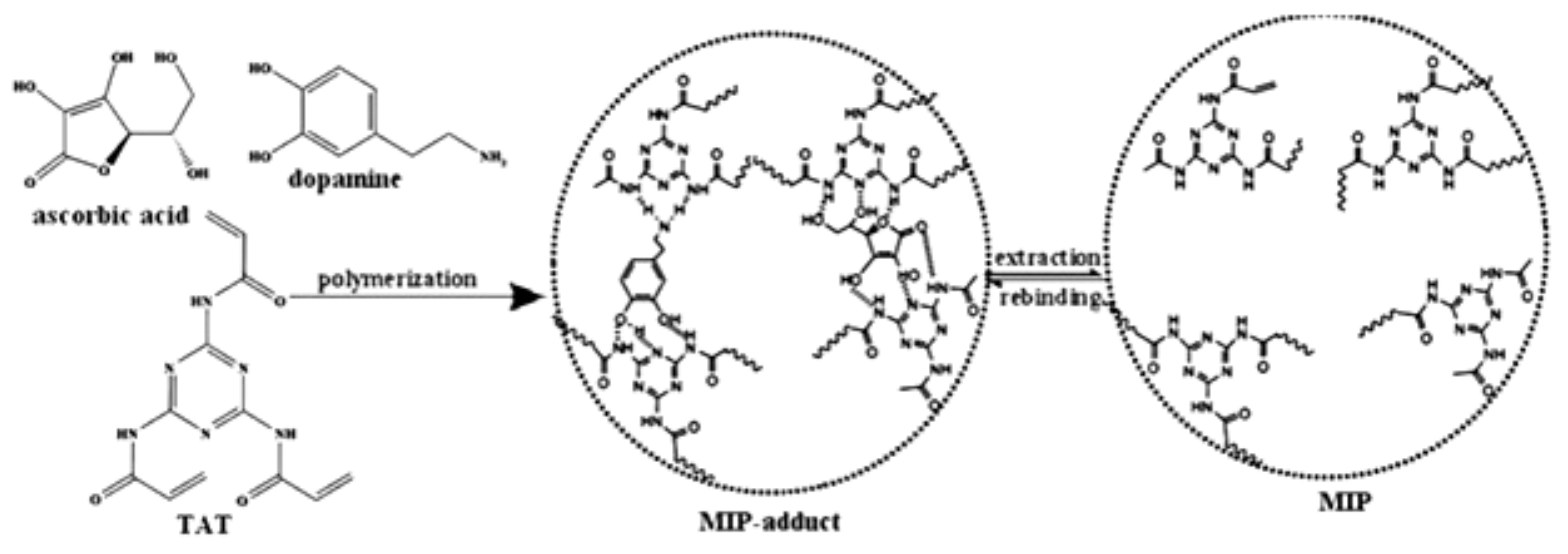

Fig. 2. MIP-carbon composite fiber imprinted with AA and DA. 
in their mixtures (AA and DA, Fig. 4 f, g). This reveals no interference of either AA or DA in their simultaneous determination. The linear relationships were found for AA and DA, in terms of calibration equations (DPCSV current, Ipc/ $\mu A$ versus concentration, $\mathrm{C} / n g m L^{-1}$ ) as given below:

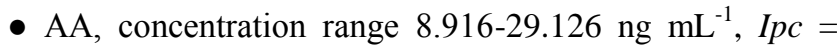
$(7.803 \pm 0.074) \mathrm{C}+(1.758 \pm 0.406), v=0.99, \mathrm{n}=6 . L O D$ $=2.635 \mathrm{ng} \mathrm{mL}^{-1}(3 \sigma, R S D=0.82 \%)$, recovery $=92.0-$ $95.1 \%$

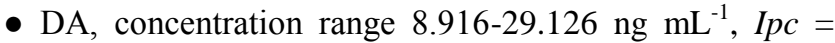
$(12.372 \pm 0.173) \mathrm{C}+(2.589 \pm 0.826), v=0.99, \mathrm{n}=6 . L O D$ $=2.247 \mathrm{ng} \mathrm{mL}^{-1}(3 \sigma, R S D=0.64 \%)$, recovery $=93.4-$ $94.7 \%$

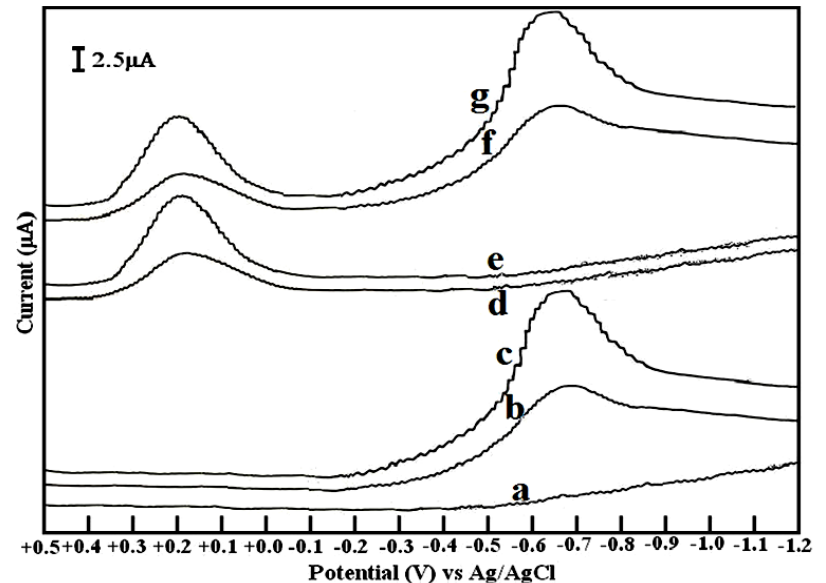

Fig. 4 DPCSV response of MIP-carbon composite fiber sensor: (a) blank, (b) and (d) $10.25 \mathrm{ng} \mathrm{mL}^{-1}$, for DA and AA, respectively; (c) and (e) 16.50 ng $\mathrm{mL}^{-1}$, for DA and AA, respectively; (f) AA and DA mixture (both $10.25 \mathrm{ng} \mathrm{mL}^{-1}$ ); (g) AA and DA mixture (both $16.50 \mathrm{ng} \mathrm{mL}^{-1}$ ).

\section{Interference studies}

The present method did not reveal any cross-reactivity with interfering species viz, cysteine, tyrosine, histidine, glycine, cystine, phenylalanine, uric acid, epinephrine, urea, and glucose. This showed a selective and specific binding between MIP and template in question, without any falsepositive contributions.

\section{Conclusion}

This work describes the use of RAFT technique to prepare MIP-carbon composite fiber sensor for the simultaneous determination of AA and DA, in aqueous samples, without any cross-reactivity and false-positives. MIP-fiber sensor has reproducible characteristics for highly sensitive measurements, without any attendant problem of surface fouling.

\section{Reference}

1. Sreenivasan, K.; J. Mater. Sci. 2007, 42, 7575.

DOI: $10.1007 / \mathrm{s} 10853-007-1625-7$

2. Suedee, R.; Srichana, T.; Chuchome, T.; Kongmark, U.; J. Chromatogr. B 2004, 811, 191. DOI: $10.1016 / j . j$ jchromb.2004.08.044

3. Andrea, P.; Miroslav, S.; Silvia, S.; Stanislav, M.; Sens. Actuators, B 2001, 76, 286.
DOI: $10.1016 / \mathrm{S} 0925-4005(01) 00586-X$

4. Mccormick, C. L.; Lowe, A. B.; Acc. Chem. Res. 2004, 37, 312. DOI: $10.1021 / \operatorname{ar} 0302484$

5. Yu, A. -M.; Chen, H. -Y.; Anal. Chim. Acta 1997, 344, 181. DOI: $10.1016 / \mathrm{S} 0003-2670(97) 00016-0$

6. Gazdik, Z.; Zitka, O.; Petrlova, J.; Adam, V.; Zehnalek, J.; Horna, A.; Reznicek, V.; Beklova, M.; Kizek, R.; Sensors 2008, 8, 7097. DOI: $10.3390 / \mathrm{s} 8117097$

7. Qian, X.; Zhang, Q.; Zhang, Y.; Tu, Y.; Anal Sci. 2010, 26, 557. DOI: $10.2116 /$ analsci.26.557

8. Legnerova, Z.; Satinsky, D.; Solich, P.; Anal. Chim. Acta 2003, 497 , 165. DOI: $10.1016 /$ j.aca.2003.07.007

9. Nezamzadeh, A.; Amini, M., K.; Faghihian, H.; Int. J. Electrochem. Sci., 2007, 2, 583.

URL: http://www.electrochemsci.org

10. Behfar, A., A.; Sadeghi, N.; Jannat, B.; Oveisi, M., R.; Iranian J. Pharm. Res. 2010, 9, 123.

URL: http://www.ijpr-online.com

11. Hubbard, K., E.; Wells, A.; Owens, T., S.; Tagen, M.; Fraga, C., H.; Stewart, C., F.; Biomed Chromatogr. 2010, 24, 626. DOI: $10.1002 / \mathrm{bmc} .1338$

12. Zhang, L.; Qvb, S.; Wanga, Z.; Cheng, J.; J. Chromatogr. B 2003, $792,381$. DOI: $10.1016 / \mathrm{S} 1570-0232(03) 00323-4$

13. Luliński, P.; Maciejewska, D.; Bamburowicz-Klimkowska, M.; Szutowski, M.; Molecules 2007, 12, 2434. DOI: $10.3390 / 12112434$

14. Mazloum-Ardakani, M.; Rajabi, H.; Beitollahi, H.; Mirjalili, B., B., F.; Akbari, A.; Taghavinia, N.; Int. J. Electrochem. Sci., 2010, 5, 147.

URL: http://www.electrochemsci.org

15. Manjunatha, J., G.; Swamy, B., E.; Gilbert, O.; Mamatha, G., P.; Sherigara, B., S.; Int. J. Electrochem. Sci., 2010, 5, 682.

URL: http://www.electrochemsci.org

16. Xu, X.; Lin, Q.; Liu, A.; Chen, W.; Weng, X.; Wang, C.; Lin, X.; Chem. Pharm. Bull. 2010, 58, 788. DOI: $10.1248 / \mathrm{cpb} .58 .788$

17. Habibia, B.; Pournaghi-Azar, M., H.; Electrochim. Acta 2010, 55, 5492. DOI: $10.1016 /$. electacta.2010.04.052

18. Tashkhourian, J.; Nezhad, M., R., H.; Khodavesi, J.; Javadi, S.; J. Elecroanal. Chem. 2009, 633, 85. DOI: $10.1016 / \mathrm{j}$.jelechem.2009.04.028

19. Prasad, B. B.; Madhuri, R.; Tiwari, M. P.; Sharma, P. S.; Biosens. Bioelectron. 2010, 25, 2140. DOI: $10.1016 /$ j.bios.2010.02.016

20. Skoog, D. A.; Holler, F.T.; Nieman, T.A.; Principles of Instrumental Analysis, 5th ed., Harcourt Brace College Publ., Orlando, FL, 1998, p. 13. 


\section{AdVAnCed Materials Letters}

\section{Publish your article in this journal}

ADVANCED MATERIALS Letters is an international journal published quarterly. The journal is intended to provide top-quality peer-reviewed research papers in the fascinating field of materials science particularly in the area of structure, synthesis and processing, characterization, advanced-state properties, and applications of materials. All articles are indexed on various databases including DOAJ and are available for download for free. The manuscript management system is completely electronic and has fast and fair peer-review process. The journal includes review articles, research articles, notes, letter to editor and short communications.

JOURNAL

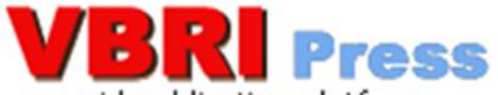

a rapid publication platform

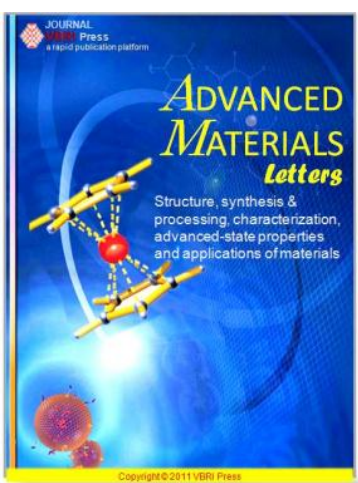

